\title{
Los aportes de la decolonialidad a las narrativas feministas en Relaciones Internacionales: aproximaciones para posibles abordajes de la cooperación internacional
}

Camila Abbondanzieri*

\section{RESUMEN}

La motivación principal que orienta el presente artículo está justificada por la necesidad de contribuir a ampliar y profundizar los objetos de estudio abordados por las perspectivas feministas en Relaciones Internacionales por dos motivos: en primer lugar, para coadyuvar al proceso de revisionismo ontológico de la disciplina inaugurado por esta clase de enfoques; $y$, en segundo lugar, para aportar a la visibilización pragmática de prácticas y dinámicas internacionales que perpetúan lógicas de subordinación y exclusión hacia distintas corporalidades con base en la matriz de dominación colonial, capitalista y patriarcal. Se parte de la premisa de que la cooperación internacional representa una interacción básica de las relaciones internacionales y que, en el marco de la literatura académica de los feminismos recibió menor atención con respecto a otro conjunto de procesos asociados con el desarrollo, los conflictos, la paz y la seguridad. En tal sentido, el propósito del presente artículo consiste en identificar los aportes de la decolonialidad a las narrativas feministas en Relaciones Internacionales a fin de contribuir a la problematización de la cooperación internacional a partir de una metodología de revisión documental de artículos y libros de autores de la disciplina de las Relaciones Internacionales. Para poder dar cuenta de ello, en la primera sección se describirá el panorama general en el que se insertaron los estudios de género en las Ciencias Sociales. Asimismo, se abordarán las condiciones de emergencia y enunciación de los feminismos hegemónicos en la disciplina de las Relaciones Internacionales y se explicitarán sus principales aportes ontológicos, epistemológicos y metodológicos. En la segunda sección, se analizará la especificidad de la narrativa situada de los feminismos decoloniales entendida como una ruptura hacia los postulados homogeneizantes y falsamente pretendidos universalistas de los feminismos hegemónicos. A partir de los lineamientos ofrecidos por las dos primeras secciones, en la tercera sección se indagará acerca de las implicancias de la cooperación internacional para los feminismos de manera general y de los feminismos decoloniales de modo particular con el propósito de dilucidar en qué medida se expresa un mecanismo perpetuador de asimetrías y de qué forma es posible lograr una superación de las lógicas patriarcales en clave emancipatoria. Finalmente, se compartirán unas breves conclusiones.

\section{Palabras clave}

Feminismos decoloniales; perspectiva feminista; teorías de relaciones internacionales; cooperación internacional; interseccionalidad; Relaciones Internacionales.

\section{TITLE}

The contributions of decoloniality to feminist narratives in International Relations: approaches for possible approaches to international cooperation

\section{Extended Abstract}

The main motivation that guides this article is the need to contribute to broadening and deepening the objects of study addressed by feminist perspectives in international relations. This is for two related reasons: firstly, to contribute to the process of ontological revisionism of the discipline that has been inaugurated by these kinds of approaches; and, secondly, to contribute to the visibility of international practices and dynamics that perpetuate logics of subordination and exclusion towards different corporeality based on the matrix of colonial, capitalist and patriarchal domination.

DOI:

https://doi.org// 0.15366/relacionesinternacionales2022.49.002

Formato de citación recomendado:

ABBONDANZIERI, Camila (2022). "Los aportes de la decolonialidad a las narrativas feministas en Relaciones Internacionales: aproximaciones para posibles abordajes de la cooperación internacional”, Relaciones Internacionales, $\mathrm{n}^{\circ}$ 49, pp. 31-49.
* Camila ABBONDANZIERI, Becaria del Consejo Nacional en Investigaciones Científicas y Técnicas (CONICET).

Doctoranda en Relaciones Internacionales por la Universidad Nacional de Rosario (UNR), Magíster en Integración y Cooperación Internacional (CERIRUNR), Licenciada en Relaciones Internacionales (UNR). Contacto: cabbondanzieri@ hotmail.com

Recibido:

30.07.2021

Aceptado:

06.11 .2021 
This article departs from the premise that international cooperation is a complex and multidimensional object of study that has historically been approached by the liberal tradition of international relations (Pereyra Rodríguez, 20 I4). Gradually, different approaches to the discipline began to inquire about the motives, procedures and outcomes as a result of the growing importance that these interactions acquired as fundamental dynamics of international relations. In the framework of the academic literature on feminisms, international cooperation has received less attention with respect to another set of processes associated with development, conflicts, and peace and security. In this sense, the purpose of this article is to contribute to the problematization of international cooperation from feminist perspectives broadly, and from decolonial feminism in particular. This will be done in a systematic way based on the methodology of documentary review.

It is important to state that in the field of international relations, feminist perspectives express a "set of ideas woven around the critique of patriarchal values and dynamics on which states and global societies were structured" (Villarroel Peña, 2007, p. 66). According to the author, the new approaches implied a series of disruptions in the ontological, epistemological and methodological dimensions that, consequently, motivated the formulation of new models of knowledge production. In such context of emergence, feminist perspectives were considered dissident proposals in relation to the traditional mainstream theoretical schools because they aimed to highlight the insufficiency of paradigmatic investigations of the discipline that had been based on a partial and not neutral approach of international dynamics and practices (Villarroel Peña, 2007; Salomón, 2002).

In order to achieve the proposed aim, in the first section of this article, the general panorama in which gender studies in the Social Sciences was inserted shall be described. Likewise, the conditions of emergence and enunciation of feminisms in the discipline of international relations will be addressed, and their main ontological, epistemological and methodological contributions will be made explicit. In the second section, the specificity of the situated narrative of decolonial feminisms, understood as a disruption of the homogenizing and falsely universalistic postulates of hegemonic feminisms, will be analyzed. Based on the guidelines offered by the first two sections, the third section will inquire into the implications of international cooperation for feminisms in general, and decolonial feminisms in particular. The goal here is to elucidate to what extent a perpetuating mechanism of asymmetries is expressed, and how it is possible to overcome patriarchal logics from an emancipatory perspective. Finally, brief conclusions will be shared.

In summary, some relevant conclusions have been found through the elaboration of the article. In the first place, it is important to note that, although international cooperation does not represent the object of study most in-depth analyzed by feminist perspectives in international relations, this focus is fundamental for the same two reasons that justify this article. That is, due to the need to contribute to the ontological revisionism of the discipline, and the need to make visible the ways in which international practices and dynamics perpetuate logics of subordination and exclusion towards different corporeality based on the matrix of colonial, capitalist and patriarchal domination.

Secondly, the study of international cooperation from perspectives -like feminism- that seek to contribute to the transformation of unequal and hierarchical gender social relations is fundamental not only in academic terms, but also in a practical way. Indeed, the combination of research and political activism promulgated by feminisms should radiate in the elucidation of the practices and dynamics of international cooperation because it is precisely in this field that important opportunities can be detected to make visible and socialize conditions of existence, resistance and struggle shared between different social groups located in different places. In effect, research must be thought of as an inessential component of political practices inasmuch as it offers horizontal action guidelines, and as it contributes to identifying the perpetuation of mechanisms that reproduce asymmetric logics in an androcentric, cisgeneric and colonial way. Thirdly, intersectionality -such a significant category for feminist approaches- can become a central hermeneutical perspective to formulate instances of international cooperation that are devised in a respectful way with the particularities of local identities, interests and expectations. Likewise, it can be shown that it allows for the provision of pragmatic content to the decolonial strategy of building alliances between social groups to promote demands that were historically invisibilized.

In short, it has been demonstrated that international cooperation itself does not represent a sign of progress, evolution or emancipation. On the contrary, we state that it is a mere interaction whose content and planning must be based on the capacity of agency of local actors, and whose development must be in accordance with the particularities expressed by each social group. In addition, feminist criticisms of the ways in which international cooperation was traditionally conducted should not be limited to advocating a greater incorporation of female officials as the ultimate goal of the demands. Indeed, recovering the criticism directed towards hegemonic feminism, particular attention should be paid to promoting pinkwashing initiatives that in no way contribute to dismantling the underlying problems that emerge from the matrix of colonial, capitalist and patriarchal domination. On the contrary, it is emphasized that international cooperation should only be a possibility in so far as it is structured on the basis of the demands of the groups involved. In this sense, initiatives must be bottom-up, and should be respectful of local identities and expectations.

Lastly, we can conclude that as long as international cooperation initiatives are not structured in a situated and contextual way, it will be very unlikely that collective emancipation strategies can be articulated. Moreover, this will only contribute to reaffirming the reproductive mechanisms of gender asymmetries in the international system. Contributions from decolonial feminisms, therefore, are essential to generate limitations to the reproduction of unequal and hierarchical gender social relations both in the areas of international cooperation policy formulation, and that of theory formulation.

\section{KEYWORDS}

Decolonial feminism;feminist approach;international relations theory;international cooperation;intersectionality; International Relations. 


\section{ntroducción}

La incorporación de los estudios de género en los ámbitos académicos representa un fenómeno cercano en el tiempo debido a que su irrupción se sitúa en el marco de la segunda ola del feminismo, es decir, hacia finales de la década del sesenta del siglo pasado (Whelehan y Pilcher, 2004). A partir de ello, a lo largo de los años subsiguientes, dicho campo experimentó una trayectoria de crecimiento progresivo que se materializó en la proliferación de espacios de discusión académicos, en la inclusión del género como objeto de estudio en las distintas currículas de las carreras de Ciencias Sociales y Humanidades en el mundo anglosajón, en el incremento de publicaciones especializadas y en la multiplicación de movimientos sociales (Thorburn, 2000). De todos modos, cabe precisar que dicho recorrido estuvo condicionado por una serie de resistencias y tensiones institucionales debido a que las nuevas líneas de investigación inauguradas por esta clase de estudios implicaron el sacudimiento de bases y estructuras académicas androcentradas $y$, como sucedió específicamente con el campo de las Relaciones Internacionales, supusieron un cuestionamiento ontológico, epistemológico y metodológico a la formas y procedimientos establecidos por las corrientes mainstream de la disciplina para conocer el mundo $y$, consecuentemente, para formular teorías.

La motivación principal que orienta el presente artículo está justificada por la necesidad de contribuir a ampliar y profundizar los objetos de estudio abordados por las perspectivas feministas en Relaciones Internacionales por dos motivos: en primer lugar, para coadyuvar al proceso de revisionismo ontológico de la disciplina inaugurado por esta clase de enfoques (Youngs, 2004); y, en segundo lugar, para aportar a la visibilización pragmática de prácticas y dinámicas internacionales que perpetúan lógicas de subordinación y exclusión hacia distintas corporalidades a partir de la matriz de dominación colonial, capitalista y patriarcal'.

Se parte de la premisa de que la cooperación internacional representa una interacción básica de las relaciones internacionales y que, en el marco de la literatura académica de los feminismos ha recibido menor atención con respecto a otro conjunto de procesos asociados con el desarrollo, los conflictos, la paz y la seguridad. En tal sentido, el propósito del presente artículo consiste en identificar los aportes de la decolonialidad a las narrativas feministas en Relaciones Internacionales a fin de contribuir a la problematización de la cooperación internacional.A partir de una metodología cualitativa y mediante las técnicas de revisión documental de artículos y libros se abordarán los principales aportes teóricos de académicas de la disciplina de las Relaciones Internacionales.

Para poder dar cuenta de ello, en la primera sección se describirá el panorama general en el que se insertaron los estudios de género en las Ciencias Sociales. Asimismo, se abordarán las condiciones de emergencia y enunciación de los feminismos en la disciplina de las Relaciones Internacionales y se explicitarán sus principales aportes ontológicos, epistemológicos y metodológicos. En la segunda sección, se analizará la especificidad de la narrativa situada de los feminismos decoloniales entendida como una ruptura hacia los postulados homogeneizantes y falsamente pretendidos universalistas de los feminismos hegemónicos.A partir de los lineamientos

La aludida matriz de dominación no representa una categoría estática, sino que refiere al conjunto de opresiones que, de manera interseccional, se ejercen de manera variable y diferenciada hacia distintos grupos sociales en virtud de sus identidades raciales, religiosas, culturales, étnicas, de género, de clase, etc. 
ofrecidos por las dos primeras secciones, en la tercera sección se indagará acerca de las implicancias de la cooperación internacional para los feminismos de manera general y de los feminismos decoloniales de modo particular con el propósito de dilucidar en qué medida estas interacciones expresan mecanismos perpetuadores de asimetrías y de qué forma es posible lograr una superación de las lógicas dominantes en clave emancipatoria. Finalmente, se compartirán unas breves conclusiones.

\section{El último bastión: las perspectivas feministas en la disciplina de las Relaciones In- ternacionales}

No resulta casual que los primeros debates acerca de la incorporación de los estudios de género en las Ciencias Sociales hayan acontecido hacia mediados de la década del sesenta en el marco de disciplinas que históricamente, como las artes y la sociología, estuvieron transitadas mayoritariamente por mujeres (Whelehan y Pilcher, 2004). En efecto, el surgimiento de los estudios de género en las Ciencias Sociales, enmarcado en un contexto global signado por el activismo de los movimientos descolonizadores y por las movilizaciones en el mundo anglosajón demandando la expansión de derechos civiles, se encuentra permeado por una impronta contestataria hacia las lógicas epistémicas excluyentes ancladas en la normatividad androcéntrica y cisgenérica (Fox Keller, 2004) En base a dicha situación, los nuevos enfoques explicitaron su propósito de visibilizar los asuntos representativos e identificatorios para la cosmovisión de las diversas identidades sexogenéricas que involucraban a un variado conjunto de intereses, experiencias y afectividades que no estaban contempladas en las estructuras académicas preexistentes (Whelehan y Pilcher, 2004). En definitiva, en sus inicios, los estudios de género se constituyeron a partir de la intencionalidad de develar los mecanismos y dispositivos de poder que generan condiciones de jerarquización entre los roles de género que subsumen al desempeño de funciones secundarias y marginales a las identidades feminizadas.

Paulatinamente, aquello que había iniciado como una tendencia espontánea y poco articulada de problematización y cuestionamiento de las lógicas imperantes en los ámbitos académicos, fue consolidándose y sistematizándose como una relevante demanda de transformación social apuntalada por una praxis política militante que continúa irradiando interrogantes en múltiples niveles locales, regionales e internacionales hasta la actualidad. Sin dudas, la interrelación entre el activismo sociopolítico y la labor académica representa un aspecto fundamental para comprender el alcance de las propuestas de investigación y el horizonte de reivindicaciones formuladas desde esta clase de movimientos (Spalter-Roth y Hartmann, 1996). En definitiva, la disputa de sentidos y contenidos en los ámbitos académicos representa una tarea crucial para lograr la incorporación de saberes surgidos en los márgenes de la institucionalidad (Whelehan y Pilcher, 2004) y para apuntalar la construcción de conocimientos situados que contemplen los intereses, deseos, expectativas y experiencias de las identidades sexo-genéricas que históricamente fueron silenciadas desde la razón androcéntrica y cisgenérica.

En otro orden de cuestiones, resulta importante precisar que el entorno de enunciación en el que se originaron los primeros estudios de género representa otra cuestión fundamental que debe ser remarcada para comprender las variantes que progresivamente se fueron desar- 
rollando con el propósito de explicitar la especificidad que asumen, por una parte, los distintos modos de subyugación y dominación perpetrados desde una matriz patriarcal, capitalista y colonial hacia los diferentes grupos sociales; $y$, por otra parte, las diversas formas de subsistencia y resistencia generadas desde los grupos sociales marginados, excluidos y discriminados en razón de su identidad sexo-genérica. De hecho, el escenario de emergencia de los estudios de género en el mundo anglosajón hacia finales de la década del sesenta, implicó que, en un primer momento, las demandas estuvieran enfocadas en la visibilización de problemáticas que afectan a un conjunto particular y acotado de mujeres - particularmente a aquellas de clases medias con trayectos formales de educación - desde la perspectiva de los feminismos liberales o institucionales. Ciertamente, las temáticas y categorías impulsadas por tales enfoques resultaban excluyentes para un amplio conjunto de identidades sexo-genéricas que, además de no estar representadas por el concepto liberal de "mujer", reivindicaban una situación de mayor opresión y vulnerabilidad causada por la interseccionalidad de discriminaciones solapadas percibidas en términos de clase, sexualidad, etnia y religión (Carastathis, 2014; Crenshaw, 1990).

A pesar de la pluralidad de representaciones que pueden detectarse en el marco de los estudios de género en base a la identificación de distintas matrices de dominación y de diversos modos de resistencia, el establecimiento de una base común de cuestionamientos onto-epistémicos y metodológicos relacionados con la disputa de la "objetividad, los criterios de verificación, la subjetividad y la reflexividad” (Goldsmith, 2002 cit. en Sosa Sánchez, 2008, p. 63) supone un hecho insoslayable. En definitiva, una de las contribuciones más relevantes de la irrupción de estos estudios consiste en la "aportación política e histórica trascendental en la epistemología mundial relacionada con la conversión de las mujeres en sujetos y objetos del conocimiento" (Goldsmith, 2002 cit. en Sosa Sánchez, 2008, p. 63). De manera progresiva, las problematizaciones inauguradas por los estudios de género, canalizadas tanto en los ámbitos académicos como en el activismo político, comenzaron a replicarse en distintos campos del conocimiento y a cuestionar, de manera específica, los entornos de enunciación teóricos de las distintas disciplinas.

En lo que respecta particularmente a las Relaciones Internacionales, el encuentro entre la disciplina y los estudios de género, dentro de los que se destacan preeminentemente las perspectivas feministas, se produjo de manera tardía en relación a lo acontecido en otros campos de las Ciencias Sociales (Tickner, 2005). Los motivos que explican dicho retraso son múltiples y están vinculados, por una parte, con una hermenéutica androcéntrica y cisgenérica que tradicionalmente se posicionó como la única clave epistémica posible para abordar las dinámicas internacionales, $y$, por otra, con las resistencias institucionales manifestadas en sistemas de valores y creencias que disponían que los asuntos de política internacional representaban un campo de investigación y de agencia exclusivamente del dominio masculino. En efecto, Sylvester (1994) señaló que, desde los albores de la disciplina, el ámbito de las Relaciones Internacionales se constituyó a partir de la naturalización de la razón masculina como el único eje posible de investigación y agencia. En base a tales consideraciones, es posible evidenciar, siguiendo a Thorburn (2000), que las Relaciones Internacionales representaron el último bastión de resistencia epistémica androcéntrica y cisgenérica de las Ciencias Sociales y el último espacio en poder ser abordado mediante los "lentes de género".

Un conjunto de autoras señala que el contexto de emergencia de las perspectivas de 
género en las Relaciones Internacionales se inserta en el marco de tendencias globales asociadas con el fin de la Guerra Fría y, en términos académicos, dentro de las reconfiguraciones ontológicas, epistemológicas y metodológicas vinculadas con el cuarto debate de la disciplina ${ }^{2}$ (Narain, 2018; Villarroel Peña, 2007; Ruiz, 2005). En primer lugar, las transformaciones globales originadas a raíz de la finalización del orden internacional bipolar y de las nuevas dinámicas de vinculación y creciente interdependencia internacional emergidas al calor de la globalización de principios de la década del noventa, coadyuvaron a la progresiva incorporación de temáticas variadas en las agendas mundiales y al establecimiento de condiciones para impulsar la expansión de reivindicaciones internacionales en los principales foros mundiales relacionadas, por ejemplo, con la materia ambiental, de género y desarrollo (Villarroel Peña, 2007). En segundo lugar, el cuarto debate de las Relaciones Internacionales entre racionalistas y reflectivistas, contribuyó a generar un entorno de cuestionamiento a los modos tradicionales de construir teoría en la disciplina y a sentar las bases para la elaboración de nuevas propuestas onto-epistémicas y metodológicas. En ese sentido, las perspectivas feministas fueron consolidándose como una respuesta a las teorías mainstream consideradas “patriarcales, parroquiales y androcéntricas” (Narain, 20 I8, p. 3).

A partir de ello, y sobre todo con la publicación del volumen 17 de la revista Millenium: Journal of International Studies de 1988, comenzaron a proponerse nuevas temáticas, objetos de estudio, marcos conceptuales y problematizaciones que no habían sido consideradas por la hermenéutica androcéntrica y cisgenérica (Grecco, 2020). Al respecto, Arévalo-Robles (2010) argumenta que "el feminismo ha logrado mostrar cómo el mainstream de las relaciones internacionales es insuficiente para dar cuenta de fenómenos que tienen implicaciones en diferentes regiones del planeta y que se encuentran interconectados por una lógica sistémica” (p. 298). Con variable intensidad, las perspectivas feministas empezaron a cuestionar $y$ a desestabilizar los pilares onto-epistémicos sobre los que se había construido la disciplina de las Relaciones Internacionales desde su surgimiento. Al respecto, Grecco (2020), señala que "las autoras feministas han contribuido a consolidar el giro reflectivista y socavar la hegemonía epistémica de los positivistas, al desvelar no sólo las desigualdades de género en el sistema internacional, sino también el etnocentrismo inherente a la disciplina" (p. 142).

En el campo de las Relaciones Internacionales, las perspectivas feministas expresan un "conjunto de ideas tejidas en torno a la crítica de los valores y las dinámicas patriarcales sobre las cuales se estructuraron estados y sociedades mundiales" (Villarroel Peña, 2007, p. 66). Según la autora, los nuevos enfoques implicaron una serie de rupturas en las dimensiones ontológicas, epistemológicas y metodológicas que, consecuentemente, motivaron la formulación de nuevos modelos de producción de conocimiento en primer término dentro del mundo anglosajón. En tal contexto de emergencia, las perspectivas feministas fueron consideradas propuestas disidentes en relación a las escuelas teóricas tradicionales del mainstream debido a que apuntaban a poner en evidencia la insuficiencia de las investigaciones paradigmáticas de la disciplina que habían estado basadas en una lectura parcial y no neutral de las dinámicas y prácticas internacionales (Villarroel Peña, 2007; Salomón, 2002).

\footnotetext{
Kepa Sodupe (2002) ubica el surgimiento del "cuarto debate" en la disciplina de las Relaciones Internacionales durante la década del noventa y está protagonizado, por un lado, por las corrientes racionalistas -integrado principalmente por los enfoques neorrealistas y neoliberalesy, por otro, por las reflectivistas - conformado por un variado conjunto de propuestas vinculadas con el constructivismo, el posmodernismo, las teorías críticas, los feminismos, etc. - . Según el autor, tal debate se caracteriza por una efervescencia teórica sin precedentes en la disciplina, por la asunción de un tono netamente filosófico y por la concentración en aspectos de teoría social.
} 
Las implicancias ontológicas de la irrupción de las perspectivas de género en las Relaciones Internacionales son múltiples. Precisamente, Youngs (2004) advierte que las perspectivas feministas inauguraron un auténtico revisionismo ontológico de una disciplina que históricamente se constituyó en términos masculinizantes. En primer lugar, se destaca la significativa contribución lograda a partir de la introducción de las relaciones sociales de género como nuevas variables que deben ser abordadas a fin de lograr una comprensión cabal y concreta de las estructuras de poder político, económico y social que se ejercen tanto en los niveles locales, como regionales e internacionales y que relegan a las identidades feminizadas a ejercer roles subordinados y marginales (Tickner, 2005). En segundo lugar, los reiterados interrogantes en torno al espacio ocupado por las mujeres (Enloe, 2016; Narain, 2014; Sylvester, 1994) permitieron explorar los mecanismos y dispositivos que sistematizaron el desempeño de roles diferenciados y jerarquizados en los ámbitos públicos y privados a partir de la representación de determinadas identidades sexo-genéricas. Es decir, desde la visión de los feminismos, la priorización de los ejes ontológicos materiales o ideacionales ${ }^{3}$ entre los que han optado las teorías mainstream de las Relaciones Internacionales carece de sentido conceptual y de operatividad práctica en tanto no se cuestione la estructura subyacente de relaciones sociales que se encuentran jerarquizadas en base al género y que, como resultado, establecen posicionamientos sociales excluyentes y marginales para quienes no formen parte de la normatividad androcéntrica y cisgenérica. En definitiva, la trayectoria de revisionismo ontológico de los feminismos en Relaciones Internacionales ha implicado una doble tarea:por una parte, comportó una obligatoria revisión endogámica de las subjetividades y de los objetos de estudio considerados en la disciplina para, posteriormente, proyectar una producción de conocimiento propia de las prácticas y dinámicas internacionales en clave de género.

También, en términos epistemológicos, las rupturas y desestabilizaciones inauguradas por las perspectivas feministas en Relaciones Internacionales son significativas. La invisibilización de la agencia femenina, tanto en la práctica política como en la construcción de conocimiento, motiva un horizonte de transformación que debe ser reconocido en los ámbitos académicos (Sylvester, 1994). Según Narain (2014), los interrogantes de las investigaciones feministas son fundamentales en tanto desafían los presupuestos centrales sobre los que se cimentó la disciplina de las Relaciones Internacionales y en tanto contribuyen a deconstruir sus conceptos principales. Por su parte, Villarroel Peña (2007) señala como una contribución nodal de los feminismos, la propuesta de renovación de conceptos, ideas y valores que inciden tanto en los espacios académicos como en las estructuras sociales, es decir, el cuestionamiento de los modos de producción de conocimiento androcentrados y cisgenéricos aportó una necesaria actualización y una relectura en clave de género del bagaje teórico-conceptual de las Relaciones Internacionales (Arévalo-Robles, 2010). En suma, las perspectivas feministas permiten una reconfiguración tanto en términos subjetivos como objetivos de la disciplina por dos motivos: en primer lugar, porque ponen de manifiesto que la subjetividad masculina cisgenérica no es la única capaz de producir conocimiento $y$, por consiguiente, que la diversidad de las identidades sexo-genéricas son igualmente indispensables para la construcción de saberes en las dinámicas internacionales; $y$, en segundo lugar, porque evidencian la inexistencia de una pretendida neutralidad de las Relaciones Internacionales al advertir que la objetividad no fue más que el pacto tácito de la matriz de dominación patriarcal, colonial y

3 Los ejes ontológicos aluden a "los referentes concretos de un discurso explicativo" (Sodupe, 2002, p. 76). Es decir, hacen referencia al contenido mismo que la disciplina pretende abordar. Mientras que, por una parte, la ontología material hace referencia al estudio de las cuestiones concretas expresadas en la realidad — recursos, capacidades, etc.—, la ontología ideacional se enfoca en el análisis de los valores, ideales, etc. 
capitalista de la razón androcentrada y cisgenérica sobre la capacidad epistémica de las diversas identidades sexogenéricas.

Finalmente, en lo concerniente a las implicancias metodológicas, las perspectivas feministas se aproximaron, en términos generales, a las proposiciones del mayor conjunto de enfoques aglutinados bajo la rúbrica del reflectivismo en el marco del cuarto debate. Según Tickner (2005), justamente el abordaje metodológico racionalista fue lo que definió a las investigaciones de las Relaciones Internacionales desarrolladas en el mundo anglosajón desde los albores de la disciplina y, por tal motivo, es necesario provocar una ruptura para dar lugar a nuevas formas de producción de conocimiento que reflejen los intereses, expectativas, deseos y experiencias de las diversas identidades sexogenéricas. En efecto, las perspectivas feministas rechazan la naturalización de los métodos científicos racionalistas que dominaron en la disciplina y, en oposición, promueven pluralismos metodológicos que expresen la especificidad y diversidad de efectos que provocan las jerárquicas y desiguales relaciones sociales de género. Ante los esquemas racionalistas, las perspectivas feministas priorizaron métodos propios de la hermenéutica y de la etnografía y adscribieron a los aportes centrados en la formación de subjetividades de los estudios históricos y sociológicos, por ejemplo (Tickner, 2005).

Desde su irrupción hasta la actualidad, diversas vertientes fueron conformándose dentro de la categoría más amplia de las perspectivas feministas en Relaciones Internacionales con el propósito de dilucidar tramas y estructuras de poder específicas y de explorar problemáticas particulares que trascendían las agendas propuestas por los feminismos liberales enunciados desde el mundo anglosajón. Sin pretensiones de compartir una taxonomía exhaustiva y detallada de las múltiples variedades de las perspectivas feministas en la disciplina, se mencionará sintéticamente a las principales expresiones que proliferaron dentro de las Relaciones Internacionales fundamentalmente en el mundo anglosajón y eurocéntrico con el propósito de visibilizar esquemáticamente sus propuestas de investigación y focos de atención prioritarios. De hecho, las clasificaciones que se han desarrollado para dar cuenta de tales ramificaciones son múltiples y están ordenadas a partir de criterios políticos y epistemológicos (Arévalo-Robles, 2010;Villarroel Peña, 2007; Salomón, 2002; Harding, 1986). Según Arévalo-Robles (2010), desde la taxonomía política pueden identificarse sintéticamente tres enfoques: el feminismo liberal ${ }^{4}$, el radical $^{5}$ y el crítico ${ }^{6}$. Por otra parte, de acuerdo con el criterio epistemológico de Harding (1986), es posible

4 Esta vertiente se define por su crítica a la "la evidente subrepresentación de la mujer en los ámbitos clásicos de las relaciones internacionales: fuerzas armadas, la conducción del Estado, órganos de representación” (Arévalo-Robles, 2010, p. 297). Es decir, desde este enfoque se aboga por una incorporación formal de mujeres en instituciones públicas sin cuestionar una matriz de dominación más amplia que genera opresiones interseccionales hacia otros conjuntos de mujeres.

5 Estos enfoques se caracterizan por considerar que "las relaciones internacionales son un fenómeno y un producto de la cosmovisión masculina, expresión de ello son la política exterior y las relaciones internacionales guerristas y anárquicas” (Arévalo-Robles, 20I0, p. 298).

6 Este último enfoque, “que inserta la postura de género estudiando las conexiones y funcionalidades entre el 'género' y la política internacional han mostrado cómo la relación entre lo público y lo privado han legitimado lo público a lo masculino y lo privado a lo femenino. Así, ha mostrado que el Estado y su política exterior se han formulado como lo público/masculino frente a la nación como madre/privado, creando una lógica de las relaciones internacionales basadas en la razón patriarcal y falocéntrica del conflicto global” (Arévalo-Robles, 20I0, p. 298) 
enumerar tres vertientes: el feminismo empiricista ${ }^{7}$, el de punto de vista ${ }^{8}$ y el posmoderno ${ }^{9}$

En suma, es posible advertir que las perspectivas feministas en las Relaciones Internacionales, a pesar de compartir como punto de partida la identificación de la desigualdad material y social causada por el simple hecho de expresar una identidad sexogenérica (Whelehan y Pilcher, 2004), representan un variado conjunto de posicionamientos onto-epistémicos y políticos que se estructuran en base a la detección de matrices de dominación patriarcal, capitalista y colonial que son variables y que generan condiciones de opresión y subyugación diferenciadas para las distintas identidades. A raíz de tales consideraciones, se fueron desarrollando líneas de investigación específicas de acuerdo con las variables contextuales y coyunturales que se manifiestan en distintos ámbitos geográficos y temporales.

Es decir, las clasificaciones en base a criterios políticos o epistemológicos aludidas representan una herramienta útil para esquematizar las propuestas surgidas en el mundo anglosajón y eurocéntrico, pero no resultan suficientes para expresar la especificidad de las representaciones, luchas, resistencias y demandas de las diversas identidades sexogenéricas que fueron emergiendo en distintas latitudes del planeta. Por lo tanto, debe remarcarse que, en las Ciencias Sociales de manera general y en las Relaciones Internacionales de modo particular, es preciso hacer alusión a "los feminismos" para dar cuenta de la pluralidad de expresiones y perspectivas teóricas que se desprenden de esta clase de estudios a partir de los condicionantes propios materializados en cada región para cada conjunto de identidades sexogenéricas.

\section{Las narrativas situadas del feminismo decolonial en las Relaciones Internacionales}

Ante las limitaciones evidenciadas en términos de representatividad subjetiva y objetiva de las perspectivas feministas enunciadas desde el mundo anglosajón para expresar las demandas, expectativas y reivindicaciones de las problemáticas específicas que perciben las diversas identidades sexo-genéricas en distintas latitudes del mundo, fueron conformándose novedosas líneas de investigación que plantearon una desestabilización a las narrativas surgidas en territorios que se desempeñaron históricamente como potencias coloniales como Estados Unidos Gran Bretaña y Francia (Grecco, 2020). En esa línea, de manera progresiva comenzaron a proliferar en los espacios académicos del Sur global perspectivas epistemológicas y abordajes metodológicos vinculados a los feminismos decoloniales que, por una parte, cuestionan la producción de conocimiento que reproduce ideas dominantes y androcéntricas de los asuntos internacionales y de las políticas globales desde una matriz hegemónica eurocéntrica (Icaza, 2017); y, por otra, resaltan el legado de la colonización como una huella abismal que se perpetúa en estructuras y

Como explica Salomón (2002), este enfoque está basado en la creencia de que "el sexismo y el androcentrismo presentes en la investigación científica son sesgos sociales que es posible corregir mediante la estricta adhesión al método científico" (p. 34).

8 De acuerdo con este enfoque, la producción de conocimiento científico está determinada por la posición dominante en razón de la norma androcéntrica y cisgenérica y, en ese sentido, sus contribuciones no son solamente parciales, sino que son también perversas (Harding, 1986). Según Salomón (2002), "la posición subyugada de la mujer les abre la posibilidad de un conocimiento más completo y menos perverso. El punto de vista de las mujeres, por lo tanto, permite desarrollar un "punto de vista" moral y científicamente preferible para las interpretaciones y explicaciones de la naturaleza y la vida social" (p. 34).

9 Esta clase de estudios se concentran en cuestionar las bases mismas de la modernidad expresadas a partir del proyecto de la llustración y de sus sistemas de valores, creencias y conocimientos correspondientes. Desde el feminismo posmoderno, todas las categorías y conceptos teóricos están sometidos a revisión (Salomón, 2002). 
bases de dominación que se mantienen hasta la actualidad y que generan opresiones diferenciadas para quienes se ubican en el Sur Global (de Sousa Santos, 20I4).

Las principales críticas esgrimidas desde los feminismos decoloniales a las vertientes hegemónicas ligadas a la perspectiva liberal, institucional y occidentalista están orientadas a socavar los constructos conceptuales de los feminismos enunciados en el mundo anglosajón y eurocéntrico que invisibilizan las múltiples opresiones ejercidas sobre las identidades sexo-genéricas del Sur Global. Dicho de otra manera, desde estas perspectivas, se cuestiona la limitada construcción de sentidos y representaciones articuladas desde los feminismos hegemónicos que desconocen la especificidad de las subyugaciones que precisamente se originan en sus propios entornos de enunciación. En tal sentido, Villarroel Peña (2018) señala que los feminismos decoloniales "desde el punto de vista epistémico y práctico se configuran como una crítica al interior del propio movimiento feminista, al cual terminan denominando feminismo hegemónico, denunciando la manera universal de pensar la subordinación de las mujeres” (p. 106).

En la misma sintonía, Lugones (2008) manifiesta que "borrando toda historia, incluyendo la historia oral, de la relación entre las mujeres blancas y las no-blancas, el feminismo hegemónico blanco equiparó mujer blanca y mujer" (p. 95). Por su parte, Grecco (2020) añade que "las feministas decoloniales critican los feminismos hegemónicos, puesto que éstos cayeron en la trampa del discurso moderno ilustrado y universalista presente tanto en el feminismo liberal con en el feminismo radical y (neo)marxista" (p. 139). En definitiva, la propuesta de los feminismos decoloniales está fundamentada en la discusión de las narrativas epistémicas hegemónicas originadas a partir del proyecto colonizador que se conjuga con la razón androcéntrica y cisgenérica y que se ejerce sobre los cuerpos y saberes subalternizados de las diversas identidades sexo-genéricas en el Sur Global.

En otro orden de cuestiones, cabe destacar que los feminismos decoloniales mantienen diálogos con otros enfoques feministas con quienes comparten el cuestionamiento a las perspectivas feministas hegemónicas, como, por ejemplo, los feminismos negros y los feminismos postcoloniales (Villarroel Peña, 2018). En tal sentido, el concepto de "interseccionalidad" resulta un ejemplo paradigmático que ilustra la identificación teórica surgida entre las corrientes aludidas. En efecto, dicha categoría proveniente de la tradición teórica de los feminismos negros en Estados Unidos (Crenshaw, 1990), resulta un aporte clave que permite ampliar las posibilidades de conceptualizar las diversas matrices de dominación y, en tal sentido, logró generar una articulación directa con los feminismos decoloniales al evidenciar la inseparabilidad y el solapamiento de aspectos identitarios como etnia, clase y género y al contribuir a visibilizar las experiencias de una clase más amplia de identidades sexo-genéricas que no se identifican con el constructo de mujer blanca liberal (Thomas, 2020; Vélez, 2019). En tal sentido, es posible advertir que la significativa relevancia de la interseccionalidad promueve un correlato entre la labor académica con el activismo político al representar pragmáticamente una "apuesta por generar prácticas que evidencien los privilegios sin caer en la victimización” (Ruiz Trejo y Betemps, 2014, p. 174).

Respecto a sus conceptualizaciones principales, es preciso destacar que el surgimiento de los feminismos decoloniales se generó a partir de la identificación de una especificidad dada por un solapamiento de opresiones y subyugaciones que se enmarcan en el proyecto colonizador 
perpetrado por las potencias centrales del sistema internacional y que produjo efectos puntuales en la jerarquización de los roles de género en los territorios colonizados. Por lo tanto, desde esta clase particular de enfoques, se acentúan las relaciones concretas de subordinación que históricamente se configuraron desde una matriz de dominación colonial, capitalista y patriarcal y se subraya la especificidad de las formas de resistencia y de las luchas que emergen a partir de tales condicionantes (Vélez, 2019).

Retomando a los principales exponentes de los estudios decoloniales en las Ciencias Sociales en América Latina como María Lugones (2008), Aníbal Quijano (2000) y Walter Mignolo (2007), Icaza (2017) advierte que el punto de partida de la reflectividad decolonial se basa en la premisa de que no existe modernidad sin una estructura colonial que la sustente. Al respecto, la autora argumenta que "la modernidad occidental es un proyecto que se constituyó como universal en el encuentro violento con la otredad" (Icaza, 2017, p. 27). En efecto, dicho encuentro violento surgido a raíz del proyecto colonizador no solamente generó impactos en los modos de organización político, económico y social y en la jerarquización de roles sexogenéricos, sino que implicó un plan sistemático de desacreditación y desarticulación de los saberes locales que provocó un auténtico epistemicidio de la producción de conocimiento local (de Sousa Santos, 2014). Por lo tanto, las condiciones de emergencia de la epistemología feminista decolonial se constituyen a partir del reconocimiento de una subalternidad impuesta tanto en el plano político como epistémico que se expresa en las corporalidades y los saberes locales del Sur Global.

Las premisas fundamentales sobre las que se centra la hermenéutica decolonial radican en la importancia del conocimiento situado y en la develación de la inexistencia de la pretendida objetividad y racionalidad positivista esgrimida desde la epistemología eurocéntrica (Rose 1997, cit. en Icaza, 2017). En ese sentido, los objetivos de los feminismos decoloniales son al mismo tiempo académicos y políticos y consisten en poner en evidencia la "colonialidad global cuestionando las epistemologías dominantes que han jugado un rol principal en la construcción de la modernidad, a través de prácticas epistemicidas que han universalizado el saber occidental y han barbarizado los conocimientos otros" (Pizarro Gómez, 2020, p. 153). En suma, sus aportes ontológicos, epistemológicos y metodológicos residen en el reconocimiento de que la agencia feminista no puede ser impuesta desde modelos hegemónicos provenientes de estructuras permeadas por lógicas de subordinación y exclusión expresadas mediante la matriz de colonización del saber y mediante retóricas salvacionistas que, como el feminismo hegemónico, redundan en narrativas patriarcales hacia las identidades sexo-genéricas de los territorios colonizados (Bidaseca y Laba, 2016). Por el contrario, los feminismos decoloniales demuestran que la justicia social solamente puede realizarse en tanto se logre una justicia epistémica global y que tal empresa solamente puede ser apuntalada a partir del reconocimiento de la legitimidad de todos los saberes y de la importancia de la construcción situada del conocimiento (de Sousa Santos, 20I4).

Por tanto, una de las principales consideraciones que se desprenden de la narrativa situada de los feminismos decoloniales es la importancia de incluir la especificidad histórica, cultural y religiosa aun en corrientes que, como el feminismo hegemónico, se definen a partir de su papel esencialmente emancipatorio. En ese sentido, el feminismo decolonial contribuye a sentar las bases para coadyuvar a una "contestación a la normativa liberal" (Mahmood, 2006, p. 2), que combata la fijación a priori de la manera en la que deben definirse las opresiones $y$, consecuentemente, 
articularse las resistencias. Así, se pone en evidencia que, incluso en movimientos con propósitos emancipatorios, es imperiosa la inclusión de una reflexión situada que incorpore múltiples dimensiones de manera interseccional, para evitar precisamente la extrapolación acrítica de modos de resistir y existir ante los diferentes estructuras y sujetos de opresión, ya que esta transferencia implica una forma de epistemicidio al ignorar las particulares configuraciones sociales e históricas que caracterizan a cada territorio y grupo social en un espacio y tiempo determinado.

En el ámbito de la disciplina de las Relaciones Internacionales, los feminismos decoloniales han proporcionado múltiples contribuciones teóricas y prácticas que, a través de la incorporación de representaciones subjetivas y objetivas situadas, han coadyuvado a dilucidar diferentes tramas de opresión ejercidas en las diversas latitudes del Sur Global. En efecto, desde esta clase de enfoques, se resalta el hecho de que la investigación situada implica necesariamente el cuestionamiento de las estructuras globales de poder debido a que, en efecto, las formas de poder-saber-ser son esencialmente relaciones históricas de dominación producto de determinadas configuraciones del orden global (Asprella y Schulz, 2020). Tales consideraciones resultan fundamentales sobre todo para una disciplina como las Relaciones Internacionales que se constituyó precisamente a partir del estudio recurrente y transversal de las vinculaciones con la otredad y de las estructuras de poder que se generan en base a las relaciones establecidas entre diversos actores en el sistema internacional. Por lo tanto, la importancia de los estudios feministas decoloniales radica en la posibilidad de profundizar en esas relaciones que se produjeron de manera violenta y que generaron efectos concretos en la jerarquización de los roles detentados a partir del género, etnia y clase de manera diferenciada en el Sur Global y que representan asuntos relevantes que se mantienen en la práctica hasta la actualidad.

Asimismo, desde los feminismos decoloniales se puntualiza que la subjetividad de la colonialidad también opera a nivel local conjugada con la razón androcéntrica y cisgenérica. Al respecto, se afirma que la colonización de los cuerpos, mentes y territorios no concluyó con los procesos de independencia y descolonización en el Sur Global. Por el contrario, la alianza infranqueable entre la tecnología de dominación colonial con el capitalismo y el patriarcado reeditó los modos de opresión y marginación que se reiteran hasta el presente. Por tal motivo, representa una urgente tarea política y social advertir que la estructura de poder global no es una construcción a-histórica y naturalmente dada, sino que es el producto de una configuración particular desde la que se trazan las líneas abismales de la marginación y la opresión (de Sousa Santos, 2014).

El cuestionamiento de la propia disciplina de las Relaciones Internacionales, históricamente enunciada en clave masculinizante y cisgenérica en el mundo anglosajón y eurocéntrico, es parte de las reivindicaciones impulsadas por los feminismos decoloniales. Sintéticamente, los abordajes del feminismo decolonial lograron incorporar a la disciplina una narrativa situada antimperialista, anticolonial y anticapitalista (Mikkonen,2020). Como se mencionó,los propósitos de transformación del feminismo decolonial en la disciplina son tanto analíticos como empíricos ya que, además de expresarse en clave emancipatoria de las relaciones sociales, involucran una superación de las lógicas epistemicidas de los saberes nacidos en los márgenes (Mikkonen, 2020). Resumidamente, Villarroel Peña (20l8) señala que esta clase de enfoques expresan una ontología política de la dominación, una epistemología feminista decolonial y una estética decolonial. 
La incorporación de los feminismos decoloniales a las Relaciones Internacionales supone un curso de acción relevante para repensar las dinámicas y prácticas internacionales a partir de nuevas categorías y claves hermenéuticas. Fundamentalmente, dichos enfoques contribuyen a demostrar la violencia inherente en la imposición de modelos de jerarquización de los roles de género y de formas de producir conocimiento que invisibilizan las experiencias y saberes locales incluso en el marco de propuestas pretendidamente emancipatorias como las esgrimidas desde los feminismos hegemónicos. Estas consideraciones implican cuestionar el avasallamiento epistémico y político acontecido en el Sur Global mediante la razón iluminista universal (Mahmood, 2006) que representa el brazo conceptual de la opresión y la colonialidad por parte de Occidente. Así, la construcción de una "objetividad" del objeto y del sujeto de la opresión que excluya el abordaje situado, implica perpetuar una determinada configuración de la opresión tal como sucedió con los feminismos hegemónicos. Mahmood (2006) claramente ejemplifica estas reflexiones a partir de su análisis de la construcción universal del deseo $y$, en ese sentido, resulta sumamente pertinente su combate a la prescripción del feminismo hegemónico como un diagnóstico de una realidad homogénea de manera normativa.Al respecto, la autora advierte que imponer una única forma de concebir y poner en práctica la "libertad" de la expresión del deseo, forma parte de la manera en la que históricamente se avasalló a la diversidad en nombre de la "universalidad".

En suma, los feminismos decoloniales en Relaciones Internacionales asumen un rol destacado para señalar que el gran riesgo de los movimientos emancipatorios reside en la suposición $a$ priori de la definición de los sujetos de la opresión y de los modos de resistencia. Por lo tanto, la importancia de dichos enfoques en la disciplina está fundamentada por su conceptualización de la relación subjetiva de las propias identidades, intereses y experiencias como constructos resultantes de relaciones de subordinación históricamente situadas y específicas.

\section{La cooperación internacional bajo la lupa de los feminismos.Abordajes generales y especificidades decoloniales}

Tal como se mencionó previamente, las perspectivas feministas motivaron una propuesta de revisionismo ontológico en la disciplina que se materializó en una reconfiguración de los objetos de estudio y de sus respectivos abordajes metodológicos de una manera diferente a las pautas de trabajo y a las agendas de investigación establecidas por las corrientes del mainstream. En base a las diferentes posturas políticoepistemológicas de las perspectivas feministas, se comenzaron a desarrollar problematizaciones y relecturas de los objetos de estudio tradicionales de las Relaciones Internacionales y se empezaron a incorporar nuevas temáticas relevantes para las diversas identidades sexo-genéricas.

Por una parte, las perspectivas feministas destacan que los objetos de estudio tradicionalmente investigados desde las corrientes mainstream de las Relaciones Internacionales, como las interacciones básicas del conflicto y la cooperación, fueron pergeñados en clave absolutamente masculinizante y excluyeron e invisibilizaron la existencia y los variados intereses, identidades y expectativas de un conjunto de subjetividades que trascienden la normatividad androcéntrica y cisgenérica. En efecto, los primeros interrogantes de investigación feminista en el mundo anglosajón de fines de la década del ochenta estuvieron orientados a identificar los roles desempeñados 
por las mujeres en los asuntos de política internacional (Enloe, 20I6). A partir de ello, quedó de manifiesto una pauta de jerarquización de los roles de género en favor de la agencia masculina heterosexual. Consecuentemente, se señala que, para los feminismos en general, la distinción entre asuntos de alta o baja política carece de sustancia en tanto no se cuestione la estructura subyacente de dominación patriarcal que genera efectos concretos de marginación y subordinación de las diversas identidades sexo-genéricas.

En base a tales presupuestos de partida, una importante proporción de investigaciones feministas se concentraron en dilucidar la capacidad agencia específica y el rol concreto desempeñado por mujeres en temáticas relativas a variadas prácticas y dinámicas internacionales como procesos de desarrollo, situaciones de conflicto y cuestiones ligadas a la paz y a la seguridad (Youngs, 2004, p. 78; Enloe, 20 I 4;Tickner, 2005). Por su parte, las dinámicas de cooperación internacional recibieron menor atención por parte de los enfoques feministas. En efecto, tales interacciones resultaron insuficientemente exploradas y representan un promisorio campo de investigación para coadyuvar al cuestionamiento de las prácticas internacionales desde la perspectiva del revisionismo ontológico promulgada desde los feminismos en Relaciones Internacionales.

La cooperación internacional ${ }^{10}$ es un objeto de estudio complejo y multidimensional que históricamente fue abordado por la tradición liberal de las Relaciones Internacionales (Pereyra Rodríguez, 20I4). De manera progresiva, distintos enfoques de la disciplina comenzaron a indagar acerca de sus móviles, procedimientos y resultados a raíz de la creciente importancia que dichas interacciones adquirieron como dinámicas fundamentales de las relaciones internacionales. Según argumenta Mikkonen (2020), los enfoques del mainstream de la disciplina de las Relaciones Internacionales reprodujeron tres trampas epistemológicas acerca del rol de las mujeres en los procesos de cooperación internacional vinculadas con su infantilización, romantización y uni-logicidad" . Tales preconceptos contribuyeron a forjar una imagen homogénea y subordinada de las mujeres como agentes de escasa trascendencia en el devenir de los procesos de cooperación internacional y cuya agencia conducida desde la emotividad resultaba impropia para apuntalar procesos de desarrollo sólidos. Dichas conceptualizaciones invisibilizan, por una parte, la diversidad de roles y experiencias habitados por las distintas identidades sexo-genéricas, y por otra, ocultan el cuestionable desempeño de actores hegemónicos y dominantes mediante sus agencias estatales o mediante ONGs que solamente perpetúan las asimetrías existentes del sistema internacional (Villarroel Peña, 2018).

En lo que respecta a las perspectivas feministas, los primeros cuestionamientos a los modos en los que se abordó la cooperación internacional provinieron de los aportes del feminismo de punto de vista expresados por Tickner (2005) y Sylvester (1994). Tickner (2005) argumentó en su crítica al realismo, que la cooperación internacional representa un componente fundamental que, junto a la interdependencia, permiten repensar la categoría de interés nacional que tradicio-

\footnotetext{
10 La cooperación internacional representa un concepto que ha sido definido de manera sumamente variada en el marco de las Relaciones Internacionales y, al mismo tiempo, supone un campo de estudio específico dentro de la disciplina. Una definición genérica de cooperación internacional implica el "comportamiento coordinado de actores independientes y posiblemente egoistas que comporta beneficios para todos ellos” (Paulo, 20I4, p. 2).

I Según la autora, la infantilización alude a la falsamente instaurada necesidad de una asistencia salvacionista dirigida hacia las mujeres que ejercen roles pasivos, secundarios y subalternizados; de manera complementaria, la romantización, a la representación de mujeres como agentes exóticos que no pueden valerse por sí mismas; $y$, finalmente, la uni-logicidad, a la incorrecta percepción de que los grupos sociales operan bajo una estructura homogénea de demandas, deseos, intereses y expectativas (Mikkonen, 2020).
} 
nalmente fue definida en términos de poder desde una hermenéutica masculinizante (Villarroel Peña, 2007). Asimismo, la autora advierte que en el mundo contemporáneo las relaciones internacionales no pueden conducirse únicamente desde la lógica del interés y del poder, sino que se requiere de vinculaciones entendidas en clave cooperativa como condición para propiciar una transformación social respetuosa de las diversas identidades sexo-genéricas (Tickner, 2005).

Sylvester (1994) introdujo la noción de cooperación empática como una metodología para apuntalar la transversalización de las problemáticas de género en las diversas dimensiones de las relaciones sociales y de las vinculaciones internacionales, y como método para repensar las prácticas de producción de conocimiento. La autora define a la cooperación empática como un "proceso de desplazamiento de posiciones que ocurre cuando se toman en cuenta seriamente las preocupaciones, miedos y agendas de quienes estuvieron excluidos de la construcción de teoría social" (Sylvester, 1994, p. 317). En consecuencia, la autora afirma la necesidad de incorporar "métodos que cuestionen todos los lugares que ocupa la teoría, desarmándolos y provocando desplazamientos o movilidad en el conocimiento que permitan generar caminos cooperativos para reinterpretar el campo disciplinar" (Sylvester, 1994, p. 3 17). De esta forma, la cooperación empática representa una auténtica redefinición epistemológica y metodológica de los modos de elaborar teorías en la disciplina y se conjuga con una apuesta por transformar en la praxis las vinculaciones sociales. Se trata de una propuesta pragmática para socavar la noción según la cual el ámbito público en el que se dirimen los asuntos de política internacional está ordenado por la razón y la lógica masculinizante, mientras que, el privado está ligado a los asuntos domésticos conducidos por las mujeres desde un criterio de emotividad y subordinación. Mediante la proposición de la cooperación empática, Sylvester (1994) fomenta el cuestionamiento de los órdenes y las lógicas que se presentaron como naturalmente dados y, como contrapartida, demuestra su construcción no neutral permeada por una fuerte carga política que pretende jerarquizar las posiciones sociales con base en el género (Butler, 1990).

Desde los feminismos decoloniales, se entiende como supuesto de partida que la cooperación internacional, como cualquier interacción impulsada por diversos actores internacionales, no puede indagarse sin considerar su contexto de enunciación y el rol desempeñado por los agentes involucrados en tales prácticas. Tradicionalmente, la cooperación internacional representó una herramienta de política exterior que los actores colonizadores planificaron para mantener sus posiciones dominantes y sus intereses en sus ex territorios coloniales tras los procesos de descolonización (Domínguez y Lucatello, 2018). El contenido y la sustancia de tales interacciones estuvieron diagramados en base a las estructuras económicas hegemónicas presentes en las políticas de desarrollo internacional (Mikkonen, 2020) y, como resultado, arrojaron esquemas de cooperación jerárquicos, paternalistas y asimétricos que redundaron en daños materiales y simbólicos para las comunidades del Sur Global a quienes se les fue negada su capacidad de agencia en la planificación de tales iniciativas (Mikkonen, 2020).

Por lo tanto, pensar en la cooperación internacional desde un enfoque feminista decolonial, implica visibilizar la existencia de corporalidades, identidades y conocimientos específicos y situados que deben participar de manera activa en todas las fases de formulación de los proyectos (Mikkonen, 2020). En tal sentido, se promueve la planificación y construcción desde las bases, involucrando a los actores locales, a partir de la orientación dada por un conjunto definido de 
pilares y lineamientos que se pueden asociar directamente a los esquemas pertenecientes a las propuestas de la Cooperación Sur-Sur que, por ejemplo, fomentan los principios de horizontalidad, solidaridad, pertinencia, consenso, reciprocidad y responsabilidad compartida y equidad (Huitron, 2016). En efecto, la premisa desde la que se gesta la Cooperación Sur-Sur está basada en la construcción de vinculaciones diagramadas en clave no jerárquica en las que todas las partes involucradas puedan desempeñar roles asertivos a partir del reconocimiento de sus propias capacidades y posibilidades.

En definitiva, análogamente a lo que se fue detectando con respecto a la lógica de cooperación Norte-Sur, los feminismos decoloniales señalan que la cooperación internacional puede reproducir lógicas asimétricas de poder en términos patriarcales sobre las diversas identidades sexo-genéricas. De manera similar a las conclusiones que se desprenden del cuestionamiento de las lógicas de cooperación Norte-Sur, se puede evidenciar que la cooperación internacional no representa per se una práctica positiva o negativa, sino que su contenido depende del modo en el que cada iniciativa es construida y planificada. Es decir, la cooperación internacional supone solamente una interacción básica del sistema internacional que puede aportar tanto beneficios como desventajas de acuerdo con la manera en la que se diagrame y ejecute.

En base a tales consideraciones, resulta claro que el devenir de la cooperación internacional solamente puede ser transformado mediante la implementación de una metodología situada y respetuosa de las identidades locales y, para ello, los aportes de los feminismos decoloniales son cruciales. A través de un estudio etnográfico acerca de las posibilidades de transformación social desde una lógica éticamente sustentable en comunidades de mujeres rurales en Nepal, Mikkonen (2020) identifica a la reflexividad y a la acción como dos instancias pragmáticas complementarias a través de las cuales se pueden pensar esquemas solidarios de cooperación internacional. Para la autora, las prácticas que apuntalan la construcción de nuevos esquemas son el diálogo, la alianza y los intercambios (Mikkonen, 2020, p. 20). Ello permite ontológica, epistemológica y metodológicamente repensar el modo de planificar nuevas experiencias evitando la reproducción de estereotipos derivados de la matriz de dominación colonial, capitalista y patriarcal y de evadir el relativismo cultural impuesto desde los agentes colonizadores.

En suma, se infiere que la cooperación internacional en sí misma no representa un signo de progreso, de evolución o de emancipación. Se trata de una mera interacción cuyo contenido y planificación deben basarse en la capacidad de agencia de los actores locales y cuyo desarrollo debe ser acorde a las particularidades que exprese cada grupo social de manera situada. Por otra parte, las críticas de los feminismos hacia los modos en los que fue conducida tradicionalmente la cooperación internacional no deben limitarse a abogar por una mayor incorporación de funcionarias como la meta última de las reivindicaciones. En efecto, recuperando los cuestionamientos esgrimidos hacia el feminismo hegemónico, se debe otorgar particular atención a la promoción de iniciativas de pinkwashing ${ }^{12}$ que en ninguna medida contribuyen a desarticular las problemáticas de fondo que emergen de la matriz de dominación colonial, capitalista y patriarcal. Por el contrario,

12 El término pinkwashing alude al conjunto de acciones emprendidas por los Estados, organismos internacionales, ONG y demás actores internacionales que buscan apropiarse de una imagen positiva para sus propias estructuras institucionales partir del aprovechamiento de las luchas de los movimientos feministas y LGBTTIQ+ (Lind, 20I4; Puar, 20I3). Tal concepto representa solamente una demostración propagandística superficial que de ninguna manera supone un cuestionamiento de la matriz de opresión que ejerce violencias y marginaciones hacia grupos sociales vulnerabilizados. En tal sentido, puede ser considerado como una reeditada forma de violencia y dominación. 
se destaca que la cooperación internacional debe ser solamente una posibilidad que se estructure en base a las demandas situadas de las agrupaciones que así lo demanden. En tal sentido, se reitera que las iniciativas deben partir de las bases y ser respetuosas de las identidades y expectativas locales orientadas hacia el respeto de los derechos humanos fundamentales y de la sostenibilidad ambiental.

En tanto las iniciativas de cooperación internacional no sean estructuradas de manera situada y contextualizada en clave decolonial, será muy poco probable que puedan articularse estrategias de emancipación colectiva y solamente se contribuirá a reafirmar los mecanismos reproductores de asimetrías en clave de género en el sistema internacional. Por lo tanto, los aportes provenientes de los feminismos decoloniales resultan imprescindible para generar limitaciones a la reproducción de relaciones sociales de género desiguales y jerarquizadas tanto en los ámbitos de formulación de políticas de cooperación internacional como en el campo de formulación de teorías.

\section{Conclusiones}

Si bien la cooperación internacional no representó el objeto de estudio mayormente profundizado por las perspectivas feministas en las Relaciones Internacionales, su abordaje resulta fundamental por los mismos dos motivos que justifican el objetivo del presente artículo, es decir, por la necesidad, por una parte, de contribuir al revisionismo ontológico de la disciplina y, por otra, siguiendo al feminismo decolonial, de visibilizar de manera pragmática las prácticas y dinámicas internacionales que perpetúan lógicas de subordinación y exclusión hacia distintas identidades sexo-genéricas en base a la matriz de dominación colonial, capitalista y patriarcal.

El estudio de la cooperación internacional desde perspectivas emancipatorias que, como los feminismos, declaran la imperiosidad de contribuir a la transformación de las relaciones sociales de género desiguales y jerarquizadas, resulta una tarea fundamental no solamente en términos académicos, sino también pragmáticos. En efecto, la conjugación de investigación y activismo político promulgado por los feminismos debe irradiar en la dilucidación de las prácticas y dinámicas de la cooperación internacional porque precisamente en este campo pueden detectarse importantes oportunidades para visibilizar y socializar condiciones de existencia, de resistencia y de lucha compartidas entre distintos grupos sociales localizados en distintas latitudes. En efecto, la investigación debe pensarse como un componente inescindible de las prácticas políticas en cuanto ofrece pautas de acción horizontales y en tanto contribuye a identificar la perpetuación de mecanismos que supieron reproducir lógicas asimétricas en clave androcéntrica, cisgenérica y colonial.

La interseccionalidad, una categoría tan significativa para los abordajes feministas no hegemónicos, puede constituirse como una clave hermenéutica central para planificar instancias de cooperación internacional que se planteen de manera respetuosa con las particularidades de las identidades, intereses y expectativas locales. Asimismo, permite dotar de contenido a la estrategia decolonial de construir alianzas entre grupos sociales para el impulso de reivindicaciones que históricamente fueron invisibilizadas. A partir de lo expuesto en el presente artículo, se concluye que el objeto de 
estudio representado por las perspectivas feministas y la cooperación internacional ofrece sendas potencialidades y cursos de investigación que, sin dudas, contribuirán a enriquecer, a dotar de pragmatismo y a vincular a la disciplina de las Relaciones Internacionales con las reivindicaciones concretas de los movimientos feministas.

\section{Referencias}

Arévalo-Robles, G.A. (2010). Las complicidades del saber-poder en el mainstream de las relaciones internacionales: una aproximación para abrir el debate. Diálogos de saberes: investigaciones y ciencias sociales, 33, 287-299.

Asprella, E. y Schulz, J.S. (2020). Colonialidad del saber, epistemologías del sur y pensamiento decolonial: crisis y oportunidades en la configuración de un nuevo orden mundial. Cuadernos de la Facultad de Humanidades y Ciencias Sociales, 57, I77-196.

Bidaseca, K. y Laba, V.V. (2016). Feminismos y (des)colonialidad. Las voces de las mujeres indígenas del Sur. Temas de mujeres, 7 (7), 22-42.

Butler, J. (1990). Gender trouble, feminist theory, and psychoanalytic discourse. En Nicholson, L. (Ed.) Feminism/ Posmodernism (pp. 324-340). Routledge.

Carastathis, A. (20I4). The concept of intersectionality in feminist theory. Philosophy compass, 9 (5), 304-3I4.

Crenshaw, K. (1990). Mapping the margins: Intersectionality, identity politics, and violence against women of color. Stanford Law Review, 43 (6), I 24|-1299.

De Sousa Santos, B. (20I4). Epistemologies of the South: Justice against Epistemicide. Paradigm Publishers.

Domínguez, R. y Lucatello, S. (20I8). Introducción: historizando y descolonizando la cooperación internacional para el desarrollo. Revista CIDOB d'afers internacionals, I20, 7-28.

Enloe, C. (20I4). Bananas, beaches and bases. University of California Press.

Enloe, C., Lacey,A.y Gregory,T. (2016). Twenty-five years of Bananas, Beaches and Bases:A conversation with Cynthia Enloe. Journal of Sociology, 52 (3), 537-550.

Fox Keller, E. (2004). What impact, if any, has feminism had on science?. Journal of biosciences, 29 (I), 7-I 3.

Grecco, G. de L. (2020). Feminismos y género en los Estudios Internacionales. Relaciones Internacionales, 44, I 27 - I 45.

Harding, S.G. (1986). The science question in feminism. Cornell University Press.

Huitron,A. (20I6). La cooperación Sur-Sur y el reto de su cuantificación, evaluación y valoración. Iberoamerican Journal of Development Studies, 5 (I), 88-I 22.

Icaza, R. (20I7). Decolonial feminism and global politics: Border thinking and vulnerability as a knowing otherwise. En Weier, S. y Woons, M. (Eds.). Critical epistemologies of global politics (pp. 26-45). E-International Relations Publishing.

Lind, A. (20I4). "Out” in international relations: why queer visibility matters. International Studies Review, I6 (4), 60 I604.

Lugones, M. (2008). Colonialidad y género. Tabula rasa, 9, 73-I0I.

Mahmood, S. (2006). Teoria feminista, agência e sujeito liberatório: algumas reflexões sobre o revivalismo islâmico no Egipto. Etnográfica, I0 (I), I2I-I58.

Mignolo,W.D. (2007). Delinking:The rhetoric of modernity, the logic of coloniality and the grammar of de-coloniality. Cultural studies, 2 I (2-3), 449-5 I4.

Mikkonen, E. (2020). Decolonial and Transnational Feminist Solidarity: Promoting Ethically Sustainable Social Change with Women in Rural Nepalese Communities. The International Journal of Community and Social Development, $2(1), 10-28$.

Narain, S. (20|4). Gender in international relations: Feminist perspectives of J. Ann Tickner. Indian Journal of Gender Studies, 2 I (2), I79- 197.

Narain, S. (2018). Gender in international relations. Global Affairs, 3 (4-5), 42I-430.

Paulo, S. (20I4). International cooperation and development: A conceptual overview. German Development Institute/ Deutsches Institut für Entwicklungspolitik Discussion Paper, I 3, I-3 I.

Pereyra Rodríguez, S. (2014). Escuelas, Modelos Metateóricos, Enfoques Teóricos y Teorías Parciales Actuales de las Relaciones Internacionales Contemporáneas. Política Internacional Contemporánea, I, I -27.

Pizarro Gómez, S. (2020). Las Relaciones Internacionales desde los feminismos descoloniales. Una propuesta dialógica hacia una economía feminista descolonial. Relaciones Internacionales, 44, I47- 164.

Puar, J. (20I3). Rethinking homonationalism. International Journal of Middle East Studies, 45 (2), 336-339.

Quijano, A. (2000). Colonialidad del poder, eurocentrismo y América Latina. En Lander, E. (Comp.). La colonialidad del saber: eurocentrismo y ciencias sociales. Perspectivas latinoamericanas (pp. 777-832). CLACSO.

Ruiz, T. (2005). Feminist theory and international relations: the feminist challenge to realism and liberalism. Soundings Journal, I-7.

Ruiz Trejo, M.G.y Betemps, C. (20I4). Epistemologías y prácticas feministas cruzadas: las posibilidades de la traducción y la importancia decolonial. Relaciones Internacionales, 27, I69-I79. 
Salomón, M. (2002). La teoría de las relaciones internacionales en los albores del siglo XXI: diálogo, disidencia, aproximaciones. Revista electrónica de estudios internacionales, 4, 3-59.

Sodupe, K. (2002). Del tercer al cuarto debate en las relaciones internacionales. Revista Española de Derecho Internacional, 65-93.

Sosa Sánchez, I.A. (2008). Feminismo y Ciencias Sociales. Revista de Antropología y Sociología: virajes, I0, 53-69.

Spalter-Roth, R. y Hartmann, H. (1996). Small happinesses: The feminist struggle to integrate social research with social activism. Feminism and social change: Bridging theory and practice, 206-224.

Sylvester, C. (1994). Empathetic cooperation:A feminist method for IR. Millennium, 23 (2), 3I 5-334.

Thomas, K.B. (2020). Intersectionality and Epistemic Erasure:A Caution to Decolonial Feminism. Hypatia, 35 (3), 509 523.

Thorburn, D. (2000). Feminism meets international relations. SAIS Review, 20 (2), I - I0.

Tickner, J.A. (2005). Gendering a discipline: Some feminist methodological contributions to international relations. Signs: Journal of Women in Culture and Society, 30 (4), 2173-2188.

Velez, E.D. (2019). Decolonial feminism at the intersection:A critical reflection on the relationship between decolonial feminism and intersectionality. The Journal of Speculative Philosophy, 33 (3), 390-406.

Villarroel Peña, Y.U. (2007). Los aportes de las teorías feministas a la comprensión de las relaciones internacionales. Politeia, 30 (39), 65-86.

Villarroel Peña, Y.U. (2018). Feminismos descoloniales latinoamericanos: geopolítica, resistencia y Relaciones Internacionales. Relaciones Internacionales, 39, 103-II9.

Whelehan, I. y Pilcher, J. (2004). Fifty key concepts in gender studies. Sage Books.

Youngs, G. (2004). Feminist International Relations: a contradiction in terms? Or: why women and gender are essential to understanding the world "we" live in. International Affairs, 80 (I), 75-87. 


\section{RELACIONES INTERNACIONALES}

Revista académica cuatrimestral de publicación electrónica Grupo de Estudios de Relaciones Internacionales (GERI)

Universidad Autónoma de Madrid, España

https://revistas.uam.es/relacionesinternacionales

ISSN 1699 - 3950

f facebook.com/RelacionesInternacionales

3. twitter.com/RRInternacional

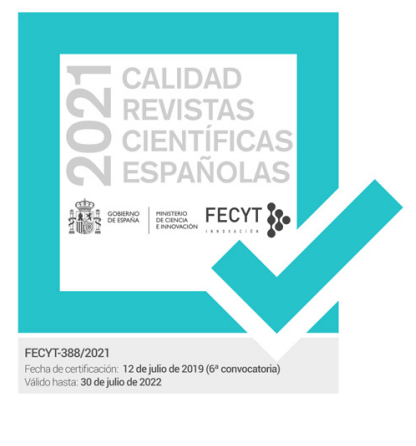

